\title{
A randomized controlled trial of brain and heart health manager-led mHealth secondary stroke prevention
}

\author{
Shasha Wang ${ }^{1 \#}$, Ying $\mathrm{Li}^{2 \#}$, Jishu Tian ${ }^{3}$, Xiaoqiong Peng ${ }^{1}$, Ling Yi ${ }^{1}$, Cuiping Du ${ }^{1}$, Changmei Feng ${ }^{1}$, \\ Chunmei Liu ${ }^{4}$, Rong Deng ${ }^{1}$, Xianju Liang ${ }^{1}$ \\ ${ }^{1}$ Department of Neurology, ${ }^{2}$ Department of Hematology, ${ }^{3}$ Department of Nursing, Chongqing Three Gorges Central Hospital, Chongqing, China; \\ ${ }^{4}$ Department of First Aid, Chongqing Three Gorges Medical College, Chongqing, China \\ Contributions: (I) Conception and design: S Wang, Y Li; (II) Administrative support: J Tian, X Peng; (III) Provision of study materials or patients: \\ None; (IV) Collection and assembly of data: C Du, C Feng (V) Data analysis and interpretation: C Liu, R Deng, X Liang; (VI) Manuscript writing: \\ All authors; (VII) Final approval of manuscript: All authors. \\ \#These authors contributed equally to this work. \\ Correspondence to: Jishu Tian, RN. Department of Nursing, Chongqing Three Gorges Central Hospital, Chongqing 404100, China. \\ Email: pengxiaoqiong1@163.com.
}

\begin{abstract}
Background: This study explores the effect of brain and heart health manager (BHHM)-led stroke secondary prevention on blood pressure, and in improving the self-management ability of stroke patients. The BHHM has not been reported.

Methods: A total of 200 stroke patients, who were discharged from our hospital, were randomized into two groups at a 1:1 ratio: intervention group and control group. Patients in the control group were followed up for six months via telephone, while patients in the experimental group were followed up for six months using the BHHM-led mHealth follow-up. The primary outcomes were systolic blood pressure (BP) and selfmanagement ability at 3, 6, 9 and 12 months, while the secondary outcomes included medication adherence, the body mass index (BMI), and blood low-density lipoprotein.
\end{abstract}

Results: The systolic BP between these two groups at four time points $\left(\mathrm{F}_{\text {time }}=8.734, \mathrm{~F}_{\text {group }}=172.075\right.$, and $\left.\mathrm{F}_{\text {interaction }}=11.363\right)$ was statistically significant $(\mathrm{P}<0.05)$. The self-health management ability at four time points during follow-up period $\left(\mathrm{F}_{\text {time }}=115.09, \mathrm{~F}_{\text {group }}=1,185.50\right.$, and $\left.\mathrm{F}_{\text {interaction }}=108.22\right)$ was also significantly different between these two groups. Furthermore, there was a statistically significant difference in compliance with medication at six months $\left(\chi^{2}=37.616, \mathrm{P}=0.000\right)$. However, after one year, there were no significant differences in $\mathrm{BMI}(\mathrm{t}=0.214, \mathrm{P}=0.644)$, total cholesterol $(\mathrm{t}=0.56, \mathrm{P}=0.837)$, and low-density lipoprotein $(\mathrm{t}=0.042$, $\mathrm{P}=0.455)$.

Conclusions: The BHHM-led mHealth follow-up is an effective method for managing BP and improving the self-care ability. Furthermore, this approach has no obvious effect on the management of BMI and blood low-density lipoprotein. A potential signal of efficacy with the intervention was observed.

Keywords: Stroke; blood pressure; brain and heart health manager (BHHM); follow-up; self-management ability

Submitted Apr 09, 2020. Accepted for publication Jul 29, 2020.

doi: $10.21037 / \mathrm{cdt}-20-423$

View this article at: http://dx.doi.org/10.21037/cdt-20-423

\section{Introduction}

Stroke recurrence increases the degree of disability and mortality of patients (1). The recurrence rate of stroke patients in the first year has reached $17.7 \%$ in China, and the accumulation rate was more than $30 \%$ in 5 years (2).
Poor self-health management ability [high systolic blood pressure (SBP), high blood lipid cholesterol, irregular administration of medications, smoking, alcoholism, and lack of exercise] has become an important factor for its recurrence (3-6). The reason why stroke recurrence is not 
uncommon is that its risk factors always exist as long as one stroke episode has occurred (7). In addition, a number of stroke patients do not have the conscience to control blood pressure or maintain a healthy lifestyle (7). Furthermore, when stroke recurs, its symptoms are worse, and its sequelae are more than the first stroke recurrence (7). Therefore, preventing stroke recurrence is very important.

Some studies have verified that specialized nurse-led interventions can greatly reduce stroke recurrence (8-10). Full-time manager-led follow-up has been widely used in the field of diabetes, which has led to good results (11). The brain and heart health manager (BHHM) is trained and assessed professionally in terms of multi-disciplinary theoretical knowledge and communication skills (12). They perform professional stroke management for chronic diseases, such as cardiovascular and cerebrovascular diseases, and are trained by the National Brain Defense Commission (12). The BHHM can provide more timely professional health interventions, allowing patients to obtain a better healthrelated knowledge (13). In previous studies, an Ambulatory Blood Pressure Monitor Mobile Health System was established to enable a patient to be aware of their risk in real-time, and this has improved the medication adherence with risk self-management (14). In the present study, the BHHM-led mobile health for secondary stroke prevention was established for post-hospital stroke patients, in order to determine its effectiveness.

We present the following article in accordance with the CONSORT reporting checklist (available at http://dx.doi. org/10.21037/cdt-20-423).

\section{Methods}

\section{Patients}

Stroke patients, who went to the Department of Neurology of an upper first-class hospital in Chongqing city between March 2019 and June 2019, and were successfully discharged, were enrolled into the present study. The study was conducted in accordance with the Declaration of Helsinki (as was revised in 2013). The study was approved by Ethics Committee of Chongqing Medical Center (No. 3) and informed consent was taken from all the patients.

Inclusion criteria: (I) residents in our district, who were $\geq 18$ years old; (II) patients with stroke defined according to the World Health Organization diagnostic criteria; (III) patients with an National Institutes of Health Stroke Scale (NIHSS) score of $\leq 4$ points, who have long-term follow-up management needs; (IV) patients with a clear mind, who are willing to participate in the present study; (V) patients who have a smart phone.

Exclusion criteria: (I) patients with serious organic diseases; (II) patients with mental diseases; (III) patients participating in other studies. The sample size calculation formula was utilized to calculate the sample size, which was finally determined to be 200 cases after considering the 10 $20 \%$ drop rate. The computer-generated random number sequence was randomly assigned to 100 cases in the control group and 100 cases in the experimental group.

\section{Intervention}

\section{Team building}

The director of the Brain Center of our hospital served as the leader of the follow-up group, and was mainly responsible for the coordination between the departments of the hospital, in order to ensure the smooth follow-up work in the hospital. The brain health manager qualifications issued by the National Health and Safety Commission Stroke Prevention and Control Engineering Committee as the deputy head of the follow-up management team are mainly responsible for each specific follow-up.

\section{Follow-up process}

After discharged from the hospital, the health information of these patients was logged in the management platform of the national stroke data center. The Bluetooth sphygmomanometer and wearable bracelet of these patients are connected to the "regional health monitoring network platform based on mobile end devices" of our hospital. At one day before the patient left the hospital, the BHHMs demonstrated the operation process, and introduce the daily maintenance precautions of mobile devices to patients, one on one. A set of mobile devices (including the Bluetooth sphygmomanometer Yuwell 680A, the Bluetooth blood glucose meter LipidEx3B, and a wearable bracelet) was given to these patients when they left the hospital. The Bluetooth sphygmomanometer monitoring module included the blood pressure monitoring record, blood pressure report, and blood pressure fluctuation data graph. The Bluetooth blood glucose meter monitoring module included the patient's blood glucose report, blood glucose monitoring record, monthly blood glucose control report, blood glucose fluctuation monitoring, blood glucose report sharing and customized blood glucose control scheme. The wearable bracelet monitoring module included the sleep 
quality analysis report, step count, mileage analysis, calorie consumption analysis and exercise target tracking. The medical terminal module included the online consultation, health education, early warning management, measurement tools and management package. The patient's Ekangwang app terminal module included the monitoring service (blood pressure data, blood pressure early warning, and intelligent bracelet), health service (health knowledge, exercise plan, diet plan, and medication reminder), hospital service (doctor's advice in the hospital, test report, and my case) and doctor service (remote guidance and online consultation). Specific operations: (I) the Bluetooth sphygmomanometer, Bluetooth blood glucose meter and wearable bracelet were connected with the information terminal of our hospital; (II) the Ekangwang app was downloaded and installed to the patient's smartphone, the Bluetooth of the phone was open, the sphygmomanometer was clicked, the blood glucose meter and bracelet connected, and the smartphone was used to search for devices and connect; (III) the data for blood pressure, blood glucose and daily calorie consumption was automatically uploaded to the medical terminal; (IV) the BHHMs certified by the National Stroke Prevention Commission analyzed the health data on the terminal, and pushed the personalized intervention scheme according to the data analysis results; $(\mathrm{V})$ health intervention measures: health education content on stroke prevention and highrisk factors was pushed daily, feedbacks on health data were reported, and the individual health education management plan was promoted; (VI) high value early warning: the BHHMs needed to communicate with patients online or by phone, when necessary, in time, in order to understand the causes of early warning, and provide solutions in time. If the data of these patients were normal for 2 weeks, the health education content pushed by the BHHMs can be reduced to 3 times/week.

\section{Evaluation index}

The primary outcome measure was SBP and selfmanagement ability at 3, 6, 9 and 12 months, while the secondary outcomes included medication adherence, body mass index (BMI) and blood low-density lipoprotein at 12 months. The self-management ability and medication adherence were utilized using the self-health management scale and medication adherence scale, respectively.

Evaluation tools: (I) the Medication Compliance Scale (15): developed by Morisky DE, which consisted of four items, with a score range of $0-4$ points, in which $0-3$ points were defined as non-compliance, while 4 points was defined as sub-table compliance. (II) The Health Self-management Ability Scale (16): this consisted of 38 items. The score ranged from 38 to 190 points, in which 38-76 points were classified as low level support, 77-152 points were classified as medium level support, and 153-190 points were classified as high level support. The higher the score, the higher the healthy self-management level.

\section{Statistical methods}

Statistical processing was performed using SPSS 20.0. All tests were bidirectional, with a significance level of $5 \%$ and a sample of 200 stroke patients. The baseline demographic/ clinical characteristics of all randomized participants were summarized using descriptive statistical methods, and baseline imbalance tests were performed on the control and experimental groups. The blood pressure measurement data of these two groups of patients at different time points were tested by multivariate test, Mauchly spherical test and internal body effect, and the relevant $95 \% \mathrm{CI}$ and $\mathrm{P}$ value were calculated. The results of the spherical test determined whether the multivariate test or the test of a patient's internal effects would be performed. If the spherical test was $\mathrm{P}<0.05$, which indicates that the spherical hypothesis was not obeyed, it was necessary to refer to the multivariate test. The multivariate test provided four methods, and the first of which is the most robust. When the four methods were inconsistent, the first one would prevail. If the spherical test was $\mathrm{P}>0.05$, the spherical hypothesis was followed, and the test of the internal effect of the subject could directly act as the observation index. The principle of the effect evaluation was to intentionally process the primary outcome data and evaluate the stability of the primary outcome. The secondary results did not consider accountability.

\section{Results}

\section{Basic information of these patients}

Table 1 presents the basic information of patients in these two groups. There were 63 males and 37 females in the control group, and these patients had an average age of $41.32 \pm 2.16$ years old. There were 58 males and 42 females in the experimental group, and their average age was $42.75 \pm 0.16$ years old. There was no statistical significance for the difference between these two groups, in terms of age, culture, disease type and stroke risk factors $(\mathrm{P}>0.05)$. 
Table 1 Demographics and primary outcomes at baseline for participants in BHHM and telephone group

\begin{tabular}{|c|c|c|c|c|}
\hline Characteristic & BHHM group & Telephone group & Significance & $P$ value \\
\hline \multicolumn{5}{|l|}{ Gender } \\
\hline Men & 58 & 63 & $0.523^{\#}$ & 0.470 \\
\hline Women & 42 & 37 & & \\
\hline Age, years & & & $0.197^{\#}$ & 0.906 \\
\hline $50-59$ & 38 & 41 & & \\
\hline $60-69$ & 31 & 30 & & \\
\hline Culture & & & $3.54^{*}$ & 0.316 \\
\hline Illiteracy & 17 & 19 & & \\
\hline Senior and above & 19 & 29 & & \\
\hline Residential area & & & $1.118^{\#}$ & 0.572 \\
\hline City & 74 & 66 & & \\
\hline Suburb & 16 & 20 & & \\
\hline Countryside & 10 & 14 & & \\
\hline Type of stroke & & & $2.524^{\#}$ & 0.112 \\
\hline Hemorrhagic & 27 & 39 & & \\
\hline Blood lipids HDL, mmol/L & $1.35 \pm 0.46$ & $1.26 \pm 0.40$ & $0.521^{\&}$ & 0.472 \\
\hline Blood lipids LDL, mmol/L & $3.79 \pm 1.52$ & $3.69 \pm 1.43$ & $0.485^{\&}$ & 0.487 \\
\hline Self-care ability & 50.08 & 50.64 & $0.29^{\#}$ & 0.590 \\
\hline Medication compliance (case) & Follow 16 & Follow 21 & $0.829^{\star}$ & 0.363 \\
\hline
\end{tabular}

${ }^{*}, \chi^{2}$; " , t value; ${ }^{\&}$, F value. HDL, high-density lipoprotein; LDL, low-density lipoprotein; SBP, systolic blood pressure; BMI, body mass index.

In the experimental group, 27 patients had hemorrhagic cerebral infarction (27\%), while 73 patients had ischemic cerebral infarction (73\%). Furthermore, in the control group, 39 patients had hemorrhagic cerebral infarction (39\%), while 61 patients had ischemic cerebral infarction $(61 \%)$. However, there were no significant difference in age, gender, culture, disease type, type of residence, SBP, medication compliance, blood lipid, BMI and self-care ability between these two groups $(\mathrm{P}>0.05)$.

\section{Changes in SBP and low-density lipoprotein from baseline to 12 months}

After the one-year follow-up period, five patients were lost to follow-up, and the rate was $2.5 \%$. Hence, a total of 195 patients were finally included. Two patients in the 


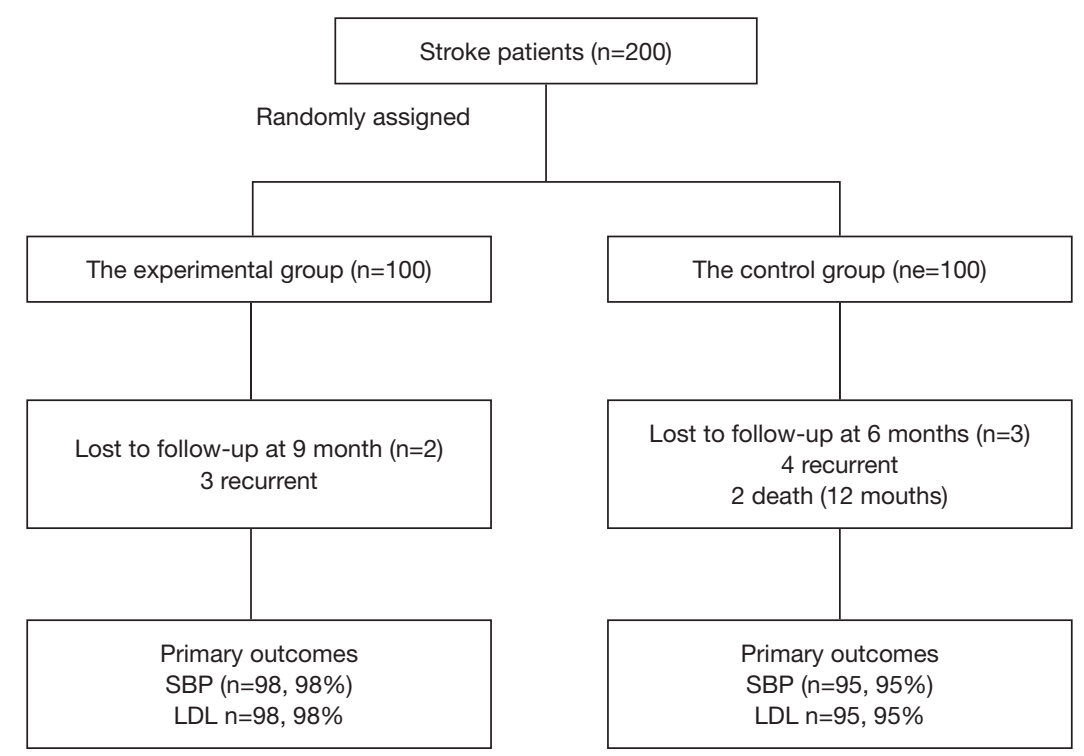

Figure 1 Study design and recruitment. LDL, low-density lipoprotein; SBP, systolic blood pressure.

Table 2 Analyses for change in systolic blood pressure and LDL from baseline to 12 months

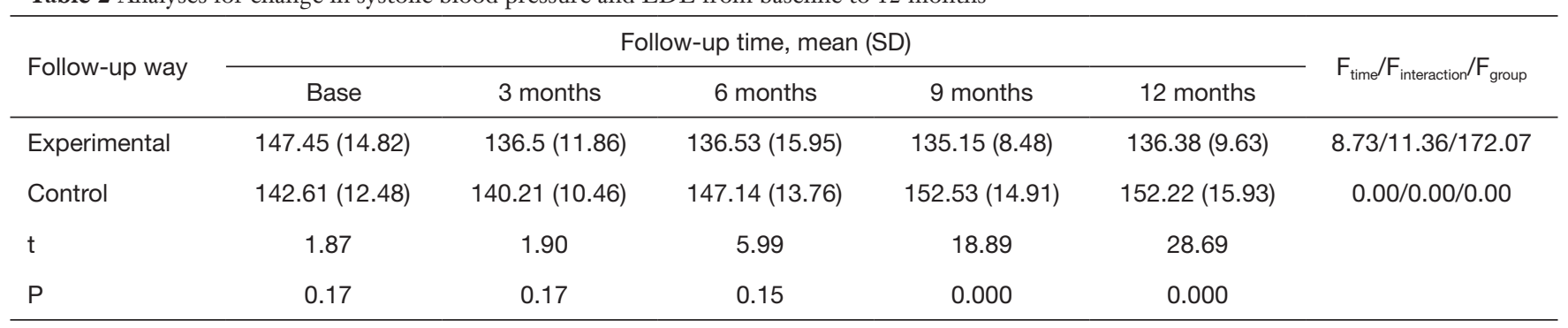

LDL, low-density lipoprotein.

experimental group were lost to follow-up due to work transfer. Hence, a total of 98 patients were included. Three patients in the control group were lost to follow-up due to work transfer and contact changes. Hence, a total of 97 patients were included (Figure 1).

As shown in Table 2, the SBP of patients in these two groups in different intervention periods are presented in Table 2. There were significant differences among the different time points before and after the follow-up $(\mathrm{F}=8.734, \mathrm{P}<0.05)$. Patients in the experimental group were able to maintain normal levels after discharge from the hospital, with minimal control at nine months. Patients in the control group maintained a normal level at only three months after discharge, and subsequently, this gradually increased. The SBP control ability of patients was significantly higher in the experimental group than in the control group. Furthermore, there were interactions before and after the follow-up, and the different follow-up modes $\left(\mathrm{F}_{\text {interaction }}=11.363, \mathrm{P}=0.000\right)$. It can be observed in Table 2 that the minimum SBP control for patients in the experimental group was nine months after discharge. The pairwise comparison revealed that the difference in SBP between the experimental group and control group was statistically significant in September after discharge.

\section{Analysis of medication compliance, BMI and low-density lipoprotein at 12 months}

As presented in Table 3, after the 1-year follow-up, there was a significant difference in medication compliance $\left(\chi^{2}=37.616\right)$ between these two groups $(\mathrm{P}<0.05)$. However, the lowdensity lipoprotein cholesterol levels $(\mathrm{t}=0.56, \mathrm{P}=0.455)$ and BMI $(t=0.214, \mathrm{P}=0.644)$ did not differ between or within the groups from baseline to 12 months. 
Table 3 Analysis of medication compliance, BMI, LDL at 12 months

\begin{tabular}{|c|c|c|c|c|c|c|c|}
\hline \multirow{2}{*}{$\begin{array}{l}\text { Intervening } \\
\text { effect }\end{array}$} & \multirow{2}{*}{$\begin{array}{c}\text { Medication } \\
\text { compliance } \\
\text { (follow/case/ } \\
\text { rate) (a year } \\
\text { later) }\end{array}$} & \multicolumn{3}{|c|}{ BMI } & \multicolumn{3}{|c|}{ LDL } \\
\hline & & Baseline & A year later & $t / P$ & Baseline & A year later & $t / P$ \\
\hline Experimental & 78 (78\%) & $25.45 \pm 2.62$ & $21.50 \pm 2.29$ & $11.34^{\#} / 0.00$ & $3.79 \pm 1.52$ & $4.85 \pm 0.86$ & $-7.03^{\#} / 0.00$ \\
\hline$P$ & 0.000 & & 0.644 & & & 0.455 & \\
\hline
\end{tabular}

${ }^{*}, \chi^{2}$; " , t value. LDL, low-density lipoprotein; BMI, body mass index.

Table 4 Analyses for change in self-care ability from baseline to 12 months

\begin{tabular}{|c|c|c|c|c|c|c|}
\hline Follow-up way & \multicolumn{5}{|c|}{ Follow-up time, mean (SD) } & $\mathrm{F}_{\text {time }} / \mathrm{F}_{\text {interaction }} / \mathrm{F}_{\text {group }}$ \\
\hline Experimental & $50.08(6.64)$ & $61.45(12.03)$ & $73.97(9.94)$ & $80.7(5.13)$ & $83.54(4.68)$ & $115.09 / 108.22 / 1,185.50$ \\
\hline Control & $50.64(6.17)$ & $53.56(4.86)$ & $51.32(6.89)$ & $59.74(7.53)$ & $49.79(6.70)$ & 0.000/0.000/0.000/0.000 \\
\hline $\mathrm{t}$ & 0.29 & 48.58 & 14.52 & 32.53 & 17.17 & \\
\hline
\end{tabular}

\section{Change in self-care ability from baseline to 12 months}

As presented in Table 4, the self-care ability of patients in the experimental group increased and reached a maximum in December. The self-care ability of patients in the control group did not significantly change before and after the intervention, and this reached the lowest level in December. There were significant differences in the two different follow-up modes $\left(\mathrm{F}_{\text {group }}=1,185.50, \mathrm{P}=0.000\right)$.

\section{Discussion}

An earlier study revealed that specialist nurses can greatly improve the effectiveness of health education (14). By managing high-risk factors and regularly pushing health education content for stroke patients using mHealth equipment, patients can observe the trend chart of their blood pressure indicators, which can better provide alerts for them. In the present study, the BHHM-led mHealth follow-up significantly improved the primary outcomes (SBP and self-care ability). This maintained the SBP at normal levels, and has a positive impact on medication compliance and self-care management ability. This may be due to the fact that BHHMs can provide more timely professional health interventions, allowing patients to obtain better health-related knowledge.

These present results are consistent with the results of previous studies. For example, an Ambulatory Blood Pressure Monitor Mobile Health System enabled a patient to be aware of their risks in real-time, which improved medication adherence with risk self-management, and the traditional telephone follow-up merely served as a reminder, with no specific intervention and lack of continuity (17). A number of studies have verified that the effectiveness of education gradually diminishes, in which medication compliance and self-management ability gradually declined with time (18). In the present study, the SBP was maintained at a normal level in the experimental group. In the control group, the SBP gradually increased at three months after discharge, predicting the key turning point for SBP intervention at three months after discharge. In the present study, the single telephone follow-up did not improve the SBP. Furthermore, the self-care ability reached a medium level at nine months, and reached the highest level at 12 months, indicating that self-care ability needs to 
be developed in a continuous health education environment.

Although there was no significant difference $(\mathrm{P}>0.05)$ in $\mathrm{BMI}$ and blood low-density lipoprotein between the two groups in the present study, the BMI before and after the intervention in the experimental group was significantly different $(\mathrm{P}<0.05)$, which indicates that there a positive impact when the BHHM-led follow-up was applied. These results may be correlated to a long-term management of BMI and blood low-density lipoprotein. A previous retrospective study indicated that stroke recurrence was $14.8 \%$ in 5.5 years using continuous follow-up and intervention, when compared to the $23.1 \%$ recurrence in the non-sustained group (19). Therefore, the study may presented with new changes if the duration of the intervention is extended.

Although the present study has achieved certain results, it was confirmed that stroke patients can benefit from the BHHM-led follow-up. However, most of the patients were urban and suburban patients. In subsequent studies, more rural patients would be included. Furthermore, the diversity of subjects would be expanded and the follow-up time would be extended, in order to verify the effectiveness of BHHM-led follow-up in remote areas.

\section{Conclusions}

The BHHM-led mHealth follow-up is an effective method for managing blood pressure and improving self-care ability. However, this has no obvious effect on the management of $\mathrm{BMI}$ and blood low-density lipoprotein. A potential signal of efficacy was observed with the intervention.

\section{Acknowledgments}

We are particularly grateful to all the people who have given us help on our article.

Funding: Chongqing Science and Health Joint Key Project: "construction and application of working mode of brain and heart health manager in the whole-course management of stroke" (No. 2019ZDXM048).

\section{Footnote}

Reporting Checklist: The authors present the study in accordance with the CONSORT reporting Checklist. Available at http://dx.doi.org/10.21037/cdt-20-423
Data Sharing Statement: Available at http://dx.doi. org/10.21037/cdt-20-423

Conflicts of Interest: All authors have completed the ICMJE uniform disclosure form (available at http://dx.doi. org/10.21037/cdt-20-423). The authors declare that they have no conflicts of interest to declare.

Etbical Statement: The authors are accountable for all aspects of the work in ensuring that questions related to the accuracy or integrity of any part of the work are appropriately investigated and resolved. The study was conducted in accordance with the Declaration of Helsinki (as was revised in 2013). The study was approved by Ethics Committee of Chongqing Three Gorges Central Hospital (No. 3), and written informed consent was obtained from all patients.

Open Access Statement: This is an Open Access article distributed in accordance with the Creative Commons Attribution-NonCommercial-NoDerivs 4.0 International License (CC BY-NC-ND 4.0), which permits the noncommercial replication and distribution of the article with the strict proviso that no changes or edits are made and the original work is properly cited (including links to both the formal publication through the relevant DOI and the license). See: https://creativecommons.org/licenses/by-nc-nd/4.0/.

\section{References}

1. Esenwa C, Gutierrez J. Secondary stroke prevention: challenges and solutions. Vasc Health Risk Manag. 2015;11:437-50.

2. Mas JL, Derex L, Guérin P, et al. Reprint of : Transcatheter closure of patent foramen ovale to prevent stroke recurrence in patients with otherwise unexplained ischaemic stroke: Expert consensus of the French Neurovascular Society and the French Society of Cardiology. Rev Neurol (Paris) 2020;176:53-61.

3. Yu F, Liu X, Yang Q, et al. Publisher Correction: Inhospital recurrence in a Chinese large cohort with acute ischemic stroke. Sci Rep 2019;9:18272.

4. da Silva CF, Schwartz J, Belli VDS, et al. Ischemic Stroke and Genetic Variants: In Search of Association with Severity and Recurrence in a Brazilian Population. J Stroke Cerebrovasc Dis 2020;29:104487. 
5. Haussen DC, Grossberg JA, Bouslama M, et al. Carotid Web (Intimal Fibromuscular Dysplasia) Has High Stroke Recurrence Risk and Is Amenable to Stenting. Stroke 2017;48:3134-7.

6. Hawkes MA, Blaginykh E, Ruff MW, et al. Long-term mortality, disability and stroke recurrence in patients with basilar artery occlusion. Eur J Neurol 2020;27:579-85.

7. Higuchi S, Kabeya Y, Matsushita K, et al. Perioperative Atrial Fibrillation in Noncardiac Surgeries for Malignancies and One-Year Recurrence. Can J Cardiol 2019;35:1449-56.

8. Kao A, Lanford J, Wong LK, et al. Do clinical nurse specialist led stroke follow-up clinics reduce post-stroke hospital readmissions and recurrent vascular events? Intern Med J 2019. doi:10.1111/imj.14707

9. Kim C, Sung J, Lee JH, et al. Clinical Practice Guideline for Cardiac Rehabilitation in Korea: Recommendations for Cardiac Rehabilitation and Secondary Prevention after Acute Coronary Syndrome. Korean Circ J 2019;49:1066-111.

10. Sharrief AZ, Hinojosa E, Cooksey G, et al. Does care in a specialised stroke prevention clinic improve poststroke blood pressure control: a protocol for a randomised comparative effectiveness study. BMJ Open 2019;9:e024695.

11. Cheng EM, Cunningham WE, Towfighi A, et al. Efficacy of a Chronic Care-Based Intervention on Secondary Stroke Prevention Among Vulnerable Stroke Survivors: A Randomized Controlled Trial. Circ Cardiovasc Qual Outcomes 2018;11:e003228.

12. Hemphill JC 3rd, Greenberg SM, Anderson CS, et al. Guidelines for the Management of Spontaneous Intracerebral Hemorrhage: A Guideline for Healthcare Professionals From the American Heart Association/

Cite this article as: Wang S, Li Y, Tian J, Peng X, Yi L, Du C, Feng C, Liu C, Deng R, Liang X. A randomized controlled trial of brain and heart health manager-led mHealth secondary stroke prevention. Cardiovasc Diagn Ther 2020;10(5):1192-1199. doi: $10.21037 /$ cdt-20-423
American Stroke Association. Stroke 2015;46:2032-60.

13. Fontaine G, Cossette S, Maheu-Cadotte MA, et al. Effect of implementation interventions on nurses' behaviour in clinical practice: a systematic review, meta-analysis and meta-regression protocol. Syst Rev 2019;8:305.

14. Forsyth F, Mant J, Taylor CJ, et al. Optimising Management of Patients with Heart Failure with Preserved Ejection Fraction in Primary Care (OPTIMISE-HFpEF): rationale and protocol for a multi-method study. BJGP Open 2019;3:bjgpopen19X101675.

15. Ganjali R, Ghorban Sabbagh M, Nazemiyan F, et al. Factors Associated With Adherence To Immunosuppressive Therapy And Barriers In Asian Kidney Transplant Recipients. Immunotargets Ther 2019;8:53-62.

16. Wang L, Tao YX, Dong XY, et al. Demographic, health behavioral, and self-management abilities associated with disease severity among patients with chronic obstructive pulmonary disease: An exploratory study. Int J Nurs Pract 2017. doi:10.1111/ijn.12509.

17. Iturralde E, Sterling SA, Uratsu CS, et al. Changing Results-Engage and Activate to Enhance Wellness: A Randomized Clinical Trial to Improve Cardiovascular Risk Management. J Am Heart Assoc 2019;8:e014021.

18. Kalter-Leibovici O, Freimark D, Freedman LS, et al. Disease management in the treatment of patients with chronic heart failure who have universal access to health care: a randomized controlled trial. BMC Med 2017;15:90.

19. Bosselmann L, Fangauf SV, Herbeck Belnap B, et al. Blended collaborative care in the secondary prevention of coronary heart disease improves risk factor control: Results of a randomised feasibility study. Eur J Cardiovasc Nurs. 2020;19:134-41. 